\title{
The JISP16 potential applied to the nucleon induced deuteron breakup process
}

\author{
Volodymyr Soloviov ${ }^{1, *}$, Jacek Golak ${ }^{1}$, Roman Skibiński ${ }^{1}$, Kacper Topolnicki ${ }^{1}$, Yuriy \\ Volkotrub $^{1}$, and Henryk Witała ${ }^{1}$ \\ ${ }^{1}$ M.Smoluchowski Institute of Physics, Jagiellonian University, Kraków, Poland
}

\begin{abstract}
The JISP16 nucleon-nucleon potential is applied to investigate the nucleon induced deuteron breakup reaction at energies $E=13$ and $65 \mathrm{MeV}$. Our study reveals that this force delivers, in general, a qualitatively similar description of the exclusive cross section for the studied reaction to the one based on the standard realistic nucleon-nucleon AV18 interaction. However, in some regions of the phase space the differential cross sections based on the JISP16 and on the AV18 forces differ by more than $100 \%$ and $50 \%$ at $\mathrm{E}=13 \mathrm{MeV}$ and $\mathrm{E}=65 \mathrm{MeV}$, respectively. Such specific parts of the phase space can be used to fine-tune the JISP16 potential parameters.
\end{abstract}

\section{Introduction}

The JISP16 potential [1] is a model of the nucleon-nucleon $(\mathrm{NN})$ interaction developed within the J-matrix inverse scattering formalism [2]. Its free parameters have been fixed by fitting to the NN phase shifts and to the energies of bound and excited states of nuclei up to ${ }^{16} \mathrm{O}$. Such a method of fixing potential parameters was chosen to study many-nucleon systems without the explicit use of many-nucleon interactions. While the JISP16 works well in the nuclear structure calculations [3,4], we have recently found [5] that the JISP16 fails in the description of some observables for the elastic nucleon-deuteron scattering process. It was deduced that the parameters of the P-wave components of the JISP16 force should be refitted. In this contribution we explore the usefulness of the nucleon-deuteron breakup process in this context.

The final exclusive kinematical configurations for the nucleon-deuteron breakup reaction are defined by five variables, e.g. the polar and azimuthal angles of momenta of two outgoing nucleons $\theta_{1}, \phi_{1}$ and $\theta_{2}, \phi_{2}$, respectively, and by the energy of one of these particles, e.g. $\mathrm{E}_{1}$. Various dynamical and kinematical aspects of the interaction dominate the breakup cross section in different parts of the five-dimensional phase space spanned by five variables mentioned above. This allows one for more systematic and detailed studies of the NN interaction, than these available e.g. in the elastic scattering process [6-9].

\section{Formalism}

We use the framework of the Faddeev equations to compute the transition amplitude and the differential cross section for the nucleon-deuteron breakup process. In this approach, the

\footnotetext{
*e-mail: volodymyr.soloviov@doctoral.uj.edu.pl
} 
Faddeev equation for an auxiliary state $T|\phi\rangle$ is the central equation to be solved. Neglecting the three-nucleon interaction it reads [6]

$$
T|\phi\rangle=t P|\phi\rangle+t P G_{0} T|\phi\rangle,
$$

where the initial state $|\phi\rangle$ is composed of the deuteron and a relative momentum eigenstate of the projectile nucleon, $P$ is a permutation operator, $G_{0}$ is the free three-nucleon propagator and $t$ is a solution of the Lippmann-Schwinger equation with a two-body interaction $V$. We solve Eq. (1) using a formalism of partial waves. For more details including the transformation of the JISP16 force from the harmonic oscillator basis to the momentum space we refer the reader to Ref. [5].

\section{Results}

To study the quality of the cross section description delivered by the JISP16 potential, we compare the JISP16 based predictions with the ones obtained using the standard AV18 potential [10]. Namely, we scan the whole phase space using a grid of 45 points for $\theta_{1}$ and $\theta_{2}, 45$ points for $\phi_{12}=\left|\phi_{2}-\phi_{1}\right|$ and a step of $0.1 \mathrm{MeV}$ along the S-curve at two incoming nucleon energies $\mathrm{E}=13$ and $\mathrm{E}=65 \mathrm{MeV}$. Note, the scan along the $\mathrm{S}$-curve is equivalent to a scan through nucleons' energies. For each kinematically complete configuration we calculate

$$
\Delta\left(\theta_{1}, \theta_{2}, \phi_{12}, E_{1}, E_{2}\right) \equiv \frac{\left(\frac{d \sigma}{d \Omega_{1} d \Omega_{2} d S}\right)^{J I S P 16}-\left(\frac{d \sigma}{d \Omega_{1} d \Omega_{2} d S}\right)^{A V 18}}{0.5\left(\left(\frac{d \sigma}{d \Omega_{1} d \Omega_{2} d S}\right)^{J I S P 16}+\left(\frac{d \sigma}{d \Omega_{1} d \Omega_{2} d S}\right)^{A V 18}\right)} .
$$

In this introductory study, instead of giving the complete exclusive information, we show in Fig. 1 maximal values of $\Delta\left(\theta_{1}, \theta_{2}\right)$ for given $\theta_{1}$ and $\theta_{2}$ taken over all remaining kinematical variables ( $\phi_{12}$ and the $\mathrm{S}$-curve). Of course some $\left(\theta_{1}, \theta_{2}\right)$ pairs are excluded by the energy and momentum conservation. Note, we also apply additional thresholds for the energy values (i.e. we take into account only configurations with $\mathrm{E}_{1} \geq 0.5 \mathrm{MeV}$ (4 MeV), $\mathrm{E}_{2} \geq 0.5 \mathrm{MeV}(4 \mathrm{MeV})$ at $\mathrm{E}=13 \mathrm{MeV}(65 \mathrm{MeV})$, respectively $)$ and for the cross sections $\left(\left(\frac{d \sigma}{d \Omega_{1} d \Omega_{2} d S}\right)^{J I S P 16}>0.4 \mathrm{fm}^{2} \mathrm{sr}^{-2} \mathrm{MeV}^{-1}\right.$ and $\left(\frac{d \sigma}{d \Omega_{1} d \Omega_{2} d S}\right)^{A V 18}>0.4 \mathrm{fm}^{2} \mathrm{sr}^{-2} \mathrm{MeV}^{-1}$, at both energies ) to avoid artificially big values of $\Delta$ and unmeasurable in practice nucleon energies.

As is seen in Fig. 1 we are able to identify regions with extreme values of $\Delta\left(\theta_{1}, \theta_{2}\right)$, what points to very different JISP16 and AV18 predictions. Here we chose particles 1 and 2 to be protons. The reader can easily find in Fig. 1 the polar angles $\left(\theta_{1}, \theta_{2}\right)$ of protons momenta for which $\Delta\left(\theta_{1}, \theta_{2}\right) \approx 100 \%$ at $\mathrm{E}=13 \mathrm{MeV}$ and $\Delta\left(\theta_{1}, \theta_{2}\right) \approx 50 \%$ at $\mathrm{E}=65 \mathrm{MeV}$.

\section{Conclusions}

The $p+d \rightarrow p+p+n$ reaction, which is known to be sensitive to the details of the nuclear forces, can be potentially used to fix parameters of the JISP16 interaction. To check this idea we have compared the JISP16 predictions for the cross section in this process with the one computed with the AV18 interaction at two energies of the incoming proton. We have found kinematical configurations for which the relative difference between both predictions amounts up to $100 \%$ and up to $50 \%$ at $\mathrm{E}=13$ and $\mathrm{E}=65 \mathrm{MeV}$, respectively. The polar angles of such configurations can be identified from the presented maps. These interesting configurations seem to be experimentally accessible. However, in most parts of the phase space the studied difference $\Delta\left(\theta_{1}, \theta_{2}\right)$ remains small, below $20 \%$. A more detailed discussion as well as a corresponding search for the analyzing powers is in preparation.

This work is a part of the LENPIC project. It was supported by the Polish National Science Centre under Grants No. 2016/22/M/ST2/00173 and 2016/21/D/ST2/01120. The numerical calculations were partially performed on the supercomputer cluster of the JSC, Jülich, Germany. 

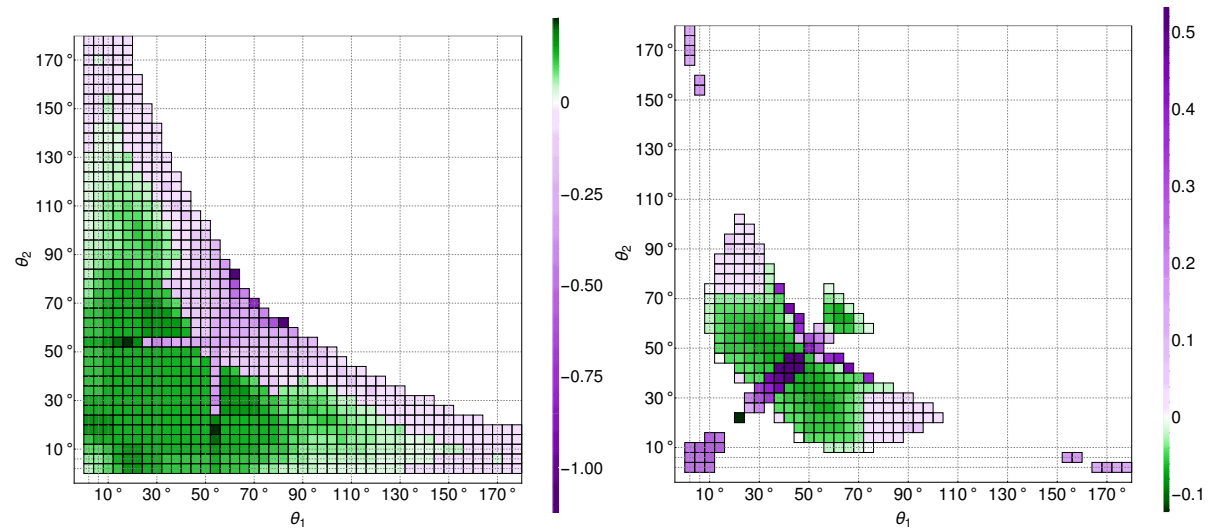

Figure 1. $\Delta\left(\theta_{1}, \theta_{2}\right)$ as a function of the polar angles $\left(\theta_{1}, \theta_{2}\right)$ of the two outgoing proton momenta at $\mathrm{E}=13 \mathrm{MeV}$ (left) and $\mathrm{E}=65 \mathrm{MeV}$ (right).

\section{References}

[1] A. M. Shirokov, J. P. Vary, A. I. Mazur, and T. A. Weber, Phys. Lett. B644, 33 (2007)

[2] A. M. Shirokov, A. I. Mazur, S. A. Zaytsev, J. P. Vary, and T. A. Weber, Phys. Rev. C70, 044005 (2004)

[3] A. M. Shirokov et al., Phys. Rev. C90, 024324 (2014)

[4] A. M. Shirokov, V. A. Kulikov, P. Maris, and J. P. Vary, in "NN and 3N Interactions", edited by L. D. Blokhintsev and I. I. Strakovsky. Nova Science, Hauppauge, NY, 2014, Chap. 8, p. 231, see https://www.novapublishers.com/catalog/product_info. php?products_id=50945

[5] R. Skibiński et al., Phys. Rev. C97, 014002 (2018)

[6] W. Glöckle et al., Phys. Rept. 274, 107 (1996)

[7] J. Kuroś-Żołnierczuk et al., Phys. Rev. C66, 024003 (2002)

[8] J. Kuroś-Żołnierczuk et al., Phys. Rev. C66, 024004 (2002)

[9] R.Skibiński, H.Witała, J.Golak, Eur. Phys. J. A30, 369 (2006)

[10] R. B. Wiringa, V. G. J. Stoks, and R. Schiavilla, Phys. Rev. C51, 38 (1995) 